\title{
Analysing Extreme Risk in the South African Financial Index (J580) using the Generalised Extreme Value Distribution
}

\author{
Delson Chikobvu , Owen Jakata * \\ Department of Mathematical Statistics and Actuarial Sciences, University of the Free State, South Africa
}

\begin{abstract}
The aim of this study is to model the probabilistic behaviour of unusually large financial losses (extreme-risk) and gains of the South African Financial Index (J580). Risk is defined as uncertainty in return in this paper. This study makes use of Extreme Value Theory (EVT) for the period years: 1995-2018 to build models that are used to estimate extreme losses and gains. The quarterly block maxima/minima of monthly returns are fitted to the Generalised Extreme Value Distribution (GEVD). Return levels (maximum loss/gain) based on the parameters from the GEVD are estimated. A comparative analysis with the Generalised Pareto Distribution (GPD) is carried out. The study reveals that EVT provides an efficient method of forecasting potentially high risks in advance. The conclusion is that analysing extreme risk in the South African Financial Index helps investors understand its riskness better and manage to reduce the risk exposure in this portfolio.
\end{abstract}

Highlights: According to the GEVD model, for one invested in the South African financial index:

i. The maximum potential losses are lower than the maximum potential gains in the short term.

ii. The maximum potential gains are lower than the maximum potential losses in the long term.

The comparative GPD model on the other hand suggests that: the maximum potential losses are greater than the maximum potential gains both in the short and long term.

Keywords block maxima/minima; extreme risk analysis; extreme value theory; generalised extreme value distribution; generalised pareto distribution; international financial crises; monthly South African Financial Index (J580); return level.

DOI: $10.19139 /$ soic-2310-5070-866

\section{Introduction}

According to [1], the world experienced a number of international financial crises since 1929, which include: a Recession in 1937-1938 in the United States of America (USA), the Brazilian stock market crash of 1971, the Black Monday crash of October 1987 in the USA, the Japanese asset price bubble from 1986 to 1991 and the Asian financial crisis of 1997. Studies on the Extreme Value Theory (EVT) have received much attention in finance after the manifestation of the international financial crises which also included the Global financial crisis (2007 -2008), European sovereign debt crisis (2010-2011) and the Chinese stock market crash (2015-2016). The catastrophic effects of these international financial crises revealed the shortcomings of financial risk models [2]. The traditional methods of estimating risk whilst assuming normality in the distribution of data fail when returns distributions are fat-tailed [3]. According to [4], EVT is a useful tool to describe the statistical properties of extreme events such as the international financial crises. Extreme equity returns require the application of the EVT methods for modelling

\footnotetext{
*Correspondence to: Owen Jakata (Email: owenjakata@ rocketmail.com). Department of Mathematical Statistics and Actuarial Sciences, University of the Free State, South Africa .
}

ISSN 2310-5070 (online) ISSN 2311-004X (print)

Copyright (C) 2020 International Academic Press 
tail-related events [5]. EVT provides a set of ready-made approaches needed for the statistical modelling of such rare events and the estimation of tail-related risk measures.

The aim of this study is to model and quantify the probabilistic behaviour of unusually large losses for one invested in the South African Financial Index (J580) using return levels, providing information to investors useful in monitoring exposure to extreme risk.

Risk can be defined as uncertainty in investment returns. Extreme risk is defined as the possibility of large losses (downside risk) and/or the possibility of large gains (upside risk). In this study the downside risk (right tail of a loss distribution) and upside risk (left tail of a loss distribution) are considered for investors with long and short positions respectively. If risk were the possibility of losses only, no one would take on the risk. It is the lure of the possibility of large gains that make investors take on risk. Risk is therefore a two edged sword with the possibility of both losses and gains. Indeed the more risk we take, the greater the possibility of large gains [6].

EVT models make reasonable predications of financial risk when the data is fat-tailed. There are two main approaches to modelling extremes. The first approach is the Block Maxima (BM) and the second is the Peaks over Threshold (PoT) approach [7]. According to [8], the main advantage in the use of the BM approach is that it avoids the problems of dependency in the data set. Dependency complicates the use of the threshold method when applying the PoT approach. The focus in this study is on extending the application of the BM approach, using the Generalised Extreme Value Distribution (GEVD), to estimate tail-related risk measures in particular for the monthly South African Financial Index (J580) return series. The estimates of extreme events provided by GEVD may underestimate the extreme events in some cases [9]. The Generalised Pareto distribution (GPD) is used to estimate extreme tail quantiles of the same returns for comparative analysis. In particular, return levels, are used as the measure of tail-related risk.

[10] confirmed that the South Africa's Johannesburg Stock Exchange (JSE) All Share Index (ALSI) is informationally efficient and that its sub-indices are not always informationally efficient. This scenario gives rise to the possibility of making excess profits/losses in the sub-indices. These sub-indices of the ALSI, such as the South African Financial Index (J580) are best modelled by the Extreme Value Distributions (EVDs) such as the GEVD and GPD.

\subsection{Statement of the Problem}

Financial markets throughout the world have been characterised by significant instabilities. The international financial crises which include: the Global financial crisis (2007 -2008), the European sovereign debt crisis (20102011) and the Chinese stock market crash (2015-2016) had a negative impact on the South African equity market. The catastrophic effects revealed the shortcomings of traditional financial risk models. The traditional methods of estimating risk whilst assuming normality in the distribution of data fail when returns distributions are fattailed. This study aims to use statistical modelling to formulate models using EVT that forecast/quantify the large losses/gains (extreme risk) in the South African Financial Index (J580). EVT models provide the framework needed for the statistical modelling and the quantification of extreme risk measures needed in portfolio risk management to minimise the negative impact of extreme events which can be very devastating.

\subsection{Justification of Study}

Investors and risk analysts are able to prepare better for extreme shocks that are associated with extreme risk behaviour of financial indices if they have access to good information about the future. EVT helps in evaluating the tail behaviour of the right tail and the left tail in the distribution of the returns data. The right and left tails are can be interchanged when a loss function is given. The statement above is equivalent to the expression: EVT helps in evaluating the tail behaviour of the return series of both the right tail of a loss function (which may be mainly losses) and left tail (which may be mainly gains). This study tries to work with loss functions were possible. 
Estimates which quantify and provide information on the maximum risk/return trade-offs needed by investors can be made when good information is available.

\subsection{Objectives of the Study}

In this study, the objectives are:

- To fit the GEVD model to the South African Financial Index (J580) returns using the block minima (maxima) method.

- To extend the application of GEVD family of distributions (Fretchet, Gumble and Weibull) in forecasting/quantifying the return levels (maximum potential loss/gain) of the South African Financial Index (J580) returns using the block minima (maxima) method.

According to [11], the Block Maxima (BM) approach can be a more efficient method than the Peaks over Threshold (PoT) method in certain instances. The practical reasons for using the BM method over the PoT method are:

- The only information available may be block maxima for a certain period with long-range historical data sets.

- If the data set is dependent and not identically distributed, the BM approach may be preferable.

- The block periods appear naturally in many situations, therefore may be easier to apply e.g. quarterly reported returns.

In this study, the BM approach uses the quarterly block periods for monthly data with their corresponding block maxima. The monthly returns are in a grouping of three, from which a selection of a maximum value within a group of three needs to done to do the data analysis.

The contribution of the study is in providing information useful to investors and risk analysts on the South African equity market and help to prepare and protect equity portfolios from future extreme losses. It differs from other studies in that it uses the South African Financial Index (J580) returns data to fit the GEVD. The study provides further empirical evidence on the usefulness of the EVT in analysing financial data in South Africa.

This study is organised as follows: section 2 presents a review of literature, section 3 presents research models, section 4 presents data analysis and discussion. Section 5 gives the conclusion and areas of further study.

\section{Review of Literature}

Extreme Value Theory is useful in many fields in the applied sciences, including insurance and finance. [3] estimated quantile risk measures for financial returns of AFAP ASURA pension fund using the Generalised Extreme Value Distribution in Uruguay. Quantiles were estimated in the tails of the of financial return series distribution based on the BM approach for a stationary time series. The main purposes of the study was to estimate the maximum loss from a portfolio. The method used Maximum Likelihood Estimation (MLE) in arriving at the parameter estimates. The return levels (maximum expected losses) for return periods 5, 10, 20 and 50 years were quantified. The researcher interpreted the return levels (maximum expected losses/gains) as a stress index to help investors protect investments against future extreme losses.

[12] modelled the distribution of extreme share return in Malaysia using the GEVD. Monthly, quarterly, half-yearly and yearly maximum returns were analysed using the BM approach. Results showed maximum returns for all selection periods were stationary. The authors concluded that yearly maxima were better for the convergence to the GEVD. The return levels that are expected to be exceeded within a certain given period are estimated at return periods of $\mathrm{T}=5,10,50$ and 100 years. The maximum return value which was recorded during the observation period is $20.174 \%$ and is expected to be exceeded after $\mathrm{T}=50$ years. This implies 
that a value of this magnitude may occur again in an average period of 50 years. Several other published papers have analysed extreme events in other areas using the GEVD, showing the importance of modelling extreme events and providing information such as return levels and periods as measures of extreme risk. [13] modelled annual maximum of daily rainfall in northern Algeria using the GEVD from 1936 to 2009 to estimate return levels at 2, 10, 20, 50 and 100 years return periods. Their results show an average of 100 years return period needed to record similar levels of $181.9 \mathrm{~mm}$ of rainfall in Algiers, $173 \mathrm{~mm}$ in Miliana and $109.54 \mathrm{~mm}$ in Oran.

[8] applied the BM approach to estimate extreme-risk using return levels at 4, 40 and 400 quarterly return periods. [7] estimated extreme-risk using return levels at 10 yearly return periods for forecasting extreme risk in the form of return levels.

According to [7], the calendar naturally suggests periods like months, quarters and years in such a way to avoid seasonal effects. Studies by [14] also show that the GEVD, which combines three different statistical distribution classes (Gumbel, Fretchet, and negative Weibull), can fit block maxima (per period maxima) of extremes of data sets with high accuracy. The same framework is useful in analysing the South African Financial Index (J580) returns data.

\section{Research Models}

The BM approach is the more traditional of the two main approaches to EVT and fits a block of minima/maxima (extreme events) in a data series of independent and identically distributed observations to the GEVD. In this study, the GEVD model is fitted to obtain parameter estimates, return levels and their related interval estimates as risk measures. The results are compared to the GPD estimates.

\subsection{Generalised Extreme Value Distribution (GEVD)}

The GEVD model is appropriate when the selected maximum observation of each period or block are from a large number of identically and independently distributed random variable [15]. According to [16], the GEVD unifies the Gumbel, Fretchet, and the negative Weibull class distributions. The combined distribution function is:

$$
G \xi, \mu, \sigma(x)=\left\{\begin{array}{lll}
\exp \left(-\left[1+\xi\left(\frac{x-\mu}{\sigma}\right)\right]^{\frac{-1}{\xi}}\right), & \text { if } & \xi \neq 0 \\
\exp \left[-\exp \left(\frac{x-\mu}{\sigma}\right)\right] & \text { if } & \xi=0
\end{array}\right.
$$

where the return on the index is $x, \mu$ is the location parameter; $\sigma$ is the scale parameter; and $\xi$ is a shape parameter or the tail index. $\xi>0$ gives rise to the Fretchet class (fat-tailed) distribution, $\xi=0$ gives rise to the Gumbel class (light tailed) distribution and $\xi<0$ gives rise to the negative Weibull class (bounded or short-tailed) distribution. The ML estimation of the unknown parameters $\mu, \sigma$ and $\xi$ are obtained by maximising the log likelihood equation with respect to the parameters [15]. The MLE is a widely used method due to its reliability and its simplicity [14]. The log likelihood of the GEVD with $n$ observations is:

$$
l_{\xi, \mu, \sigma}=-n \ln \sigma-\left(1+\frac{1}{\xi} \sum_{i=1}^{n}\left[\ln \left(1+\xi\left(\frac{x-\mu}{\sigma}\right)\right]-\sum_{i=1}^{n}\left[\left(1+\xi\left(\frac{x-\mu}{\sigma}\right)^{\frac{-1}{\xi}}\right]\right.\right.\right.
$$

Provided that $1+\xi\left(\frac{x-\mu}{\sigma}\right)>0$ for $i=1,2, \ldots \ldots \ldots$.

Where the return from period $i$ of the index is $x_{i}$. Differentiating the log of the likelihood with respect to each of the parameters gives rise to a system of equations, which yield the estimates when equated to zero.

\subsubsection{Selection of the block interval for maxima/minima returns}

Stat., Optim. Inf. Comput. Vol. 8, December 2020 
The block length is such that that an individual block maximum has a common distribution with other block maxima and converge to the GEVD [7]. To select the maxima data in this study, quarterly block intervals to monthly data were appropriate. The partitioning of the monthly data into quarterly blocks resulted in 91 blocks. There are 91 data points in each case for minima (right tail of loss function) and maxima (left tail of loss function). These are sufficient blocks to model the data using the GEVD.

\subsubsection{Modelling minima/maxima values}

In modelling the minimum values, the duality in analysis between minimum and maximum is considered. The minimum problem converts into a maximum problem by using the loss function. Heavy loss values, which were negative, become positive after multiplying by negative one. They become maxima points. According to [15], the MLE parameters of this distribution correspond exactly to those of the required GEVD for minima except the sign correction of the location parameter, which becomes negative $(-\mu)$.

Suppose $X_{1}, X_{2}, X_{n}$ is a sequence of independent and identically distributed (i.i.d) values of the index returns, the duality principle between the distributions of the minima and maxima is utilised to fit the distribution to the maximum losses(minima):

Let

$$
M_{n}=\min \left\{X_{1}, X_{2}, X_{n}\right\}
$$

be the minimum over an $n$-observations period

The minima in the block becomes

$$
\hat{M}_{n}=\max \left\{-X_{1},-X_{2},,-X_{n}\right\}
$$

when problem becomes a maxima problem: where $X_{i}$ for $i=1,2, n$ represent the equity returns from the index

For the Modelling block maxima (maximum gain), the maxima in the block is simply:

$$
M_{n}=\max \left\{X_{1}, X_{2}, X_{n}\right\}
$$

where $M_{n}$ is the maximum over an $n$-observations period. The methodology is applied to right tail, in the left tail case of the return distribution, the sign of the returns changes so that positive values correspond to losses.

\subsection{Tail-related risk measures}

Modelling of extreme events in finance is of great importance in our daily life. The issues of concern to most investors and risk analysts are the events that occur under extreme market conditions such as stock market crashes and currency crises. These events produce huge unexpected losses, which can lead to bankruptcy. Risk management in finance involves the estimation of tail-related risk measures such as Value at Risk (VaR), Expected Shortfall (ES) and the Return Levels. In this study, the GEVD gives the information to calculate the tail-related risk measures including: return levels and their related intervals.

\subsection{Return Level and Return Period for the GEVD}

When the GEVD is fitted to a dataset, it captures extreme behaviours and can describe potential losses/gains by estimating the return levels. The estimation of the return levels offers a common way to estimate the financial risk. The MLE estimates of parameters are calculated. If $G$ is the cumulative distribution of the maxima observed over successive non overlapping periods of equal length, then the return level is summarised as:

$$
R_{n}^{k}=G^{-1}\left(1-\frac{1}{k}\right)
$$

$R_{n}^{k}$, is the level expected to be exceeded in one out of $k$ periods of length $N$. 
The return period is a measure of an average period until the next maximum loss of similar magnitude in a portfolio. The level of maximum loss over that return period is the return level. The return levels for the GEVD are:

$$
R^{k}=\left\{\begin{array}{lc}
\hat{u}-\frac{\hat{\sigma}}{\xi}\left(1-\left(-\log \left(1-\frac{1}{k}\right)\right)^{-\hat{\xi}},\right. & \hat{\xi} \neq 0, \\
\hat{u}-\hat{\sigma} \log \left(-\log \left(1-\frac{1}{k}\right)\right), & \hat{\xi}=0
\end{array}\right.
$$

where $\hat{u}, \hat{\sigma}$ and $\hat{\xi}$ are the GEVD parameter estimates.

\subsection{The Peak over Threshold Method of the Generalised Pareto Distribution}

The second method used to analyse the return distribution for comparative analysis is the PoT method which extracts values that exceed a certain threshold and converge to a GPD when the threshold is sufficiently high [17] and [18].

The limiting distribution function for GPD is given by:

$$
G_{\xi, \beta}(x)=\left\{\begin{array}{lll}
1-\left(1+\frac{\xi x}{\beta}\right)^{\frac{-1}{\xi}}, & \text { if } \quad \xi \neq 0 \\
1-e^{\frac{-x}{\beta}} & \text { if } \quad \xi=0
\end{array}\right.
$$

where $x>0$ when $\xi \geq 0,0 \leq x \leq \frac{\beta}{\xi}$ when $\xi<0$ and $\beta>0$ with $\beta$ is the scale parameter and $\xi$ is the shape parameter.

\subsection{Return Level and Return Period for GPD.}

For the GPD model, the return level is explained by $R_{m}^{k}$ that defines the extreme level that is exceeded on average once every m observations.

$$
R_{m}^{k}=\hat{\mu}+\frac{\hat{\delta}}{\hat{\xi}}\left[\left(\frac{m d n_{c}}{n}\right)^{\hat{\xi}}-1\right],
$$

where $m$ is the yearly return level, $\mu$ is the threshold value, $d$ the number of observations in a year, $n_{c}$ the number of exceedances greater than $\mu$ and $n$ the total number of observations.

In finance, a return period is the average waiting period before observing the maximum potential loss/gain of the same magnitude. Therefore the return level is the value level the maximum (minimum) can reach within a return period.

\subsection{Testing for Stationarity, Normality, Heteroscedasticity and Autocorrelation}

The Augmented Dickey-Fuller (ADF) test is a test that determines whether you can conclude if a time series is stationary. In this study, the ADF tests whether the South African Financial Index (J580) returns are a stationary series. The Anderson-Darling Test is used is used to test for normality of the Index return series.. To test for the presence of heteroscedasticity in the residuals of the Index return series, the Lagrange Multiplier (LM) test for ARCH effects proposed by [19], is applied. A Box-Ljung test statistic tests if there is autocorrelation in the data set.

The LjungBox test may be defined as:

$H_{0}$ : The data are independently distributed.

$H_{1}$ : The data are not independently distributed; they exhibit auto correlation.

It tests whether the South African Financial Index (J580) return series is independently distributed. 


\subsection{Data}

The study uses the South African Financial Index (J580) data obtained (with permission) from the website iress expert: https://expert.inetbfa.com. The data consists of 272 monthly closing prices of the Index spanning the years 1995-2018. This gives rise to 91 blocks (of three) from which selection of maximum /minimum values takes place within each block. Three main, among many, sub-indices of the Johannesburg Stock Exchange All Share Index (ALSI) in South Africa(SA) based on their revenue are SA Resources, SA Financials and SA Industrials. The South African financial sector is defined as the banking, insurance and securities industries and contributes to the growth of the economy in terms of growth in assets and value added [20]. According to [21], the financial sector is responsible for a quarter of the total economic growth and employs over 220,000 people. It is important to have a financial system in which good risk management implementation results in low systemic risk.

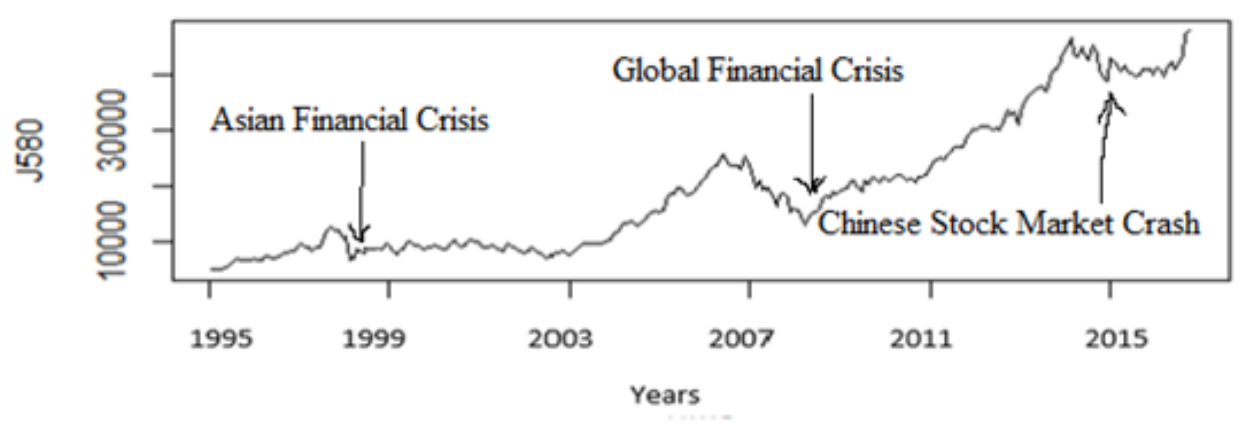

Figure 1. Time series plot of monthly values $M_{t}$ of the South African Financial Index (J580).

In Figure 1, the plot of the monthly return levels of the South African Financial Index (J580) are trending upwards from 1995 to 2018 . The international financial crises, which clearly had a negative impact on the South African equity market are: the 1997-1998 Asian financial crisis: the 2007-2008 Global financial crisis and the 2015-2016 Chinese stock market crash which all triggered the stock market crashes around the world. The international financial crises had negative effects on the South African Financial Index (J580) return levels, which are identifiable on the time series plot by sharp drops in the index level in 1997-1998, 2007-2008 and 2015-2016 respectively.

Modelling is done on the monthly log returns of the South African Financial Index (J580) using the BM approach. The BM approach fits the GEVD to quarterly block maxima to the monthly return series. Estimated parameters assist in arriving at point and interval estimates of return levels and their intervals. The monthly logreturns are:

$$
r_{t}=\ln \frac{M_{t}}{M_{t-1}}
$$

where $r_{t}$ denotes the monthly logarithmic returns in month $t, M_{t}$ represents the index value in month $t$ and $l n$ represents the natural logarithm. To model the right tail of the loss distribution (which may be mainly losses); the sign of the return data is changed such that $L_{t}=-r_{t} . L_{t}$ is the loss function. When using a loss function, the losses (minimum returns) are on the right tail and the gains (maximum returns) are on left tail of the distribution as discussed earlier.

\section{Data Analysis and Discussion}

The data was analysed in the R-programming environment using packages fExtremes, nortest, ReIns, extRemes, evir, ismev, and a TSA. 


\subsection{Testing for Stationarity}

The ADF is the test statistic (-7.3046) (lag order =6). The more negative the number, the lower the p-value. The p-value (0.01) is less than the significance level of 0.05 , we reject the null hypothesis of a unit root and conclude in favour of the alternative hypothesis that the alternate hypothesis that the monthly South African Financial Index (J580) returns data is stationary .

\subsection{Test for Normality}

The Anderson-Darling Test is used to check if the monthly South African Financial Index (J580) returns data is normally distributed, the p-value $=6.903 \mathrm{e}-08$ which is less than 0.05 , suggesting the rejection of the hypothesis of normality and concluding that the monthly data series is not normally distributed. This implies that, at the tails, the quantiles of the empirical distribution are far away from the quantiles of the normal distribution. This suggests the returns follow a fat-tailed distribution.

\subsection{Test for Heteroscedasticity}

The ARCH LM test results of monthly South African Financial Index (J580) returns indicated no presence of significant ARCH effects in the data (Chi-squared $=0.1155$, degrees of freedom $=12, \mathrm{p}$-value $=1)$. The tests performed on the standardised series and squared standardised series for conditional heteroscedasticity revealed that there is no persistence of variance and no evidence of volatility clustering in the return distribution.

\subsection{Test for Autocorrelation}

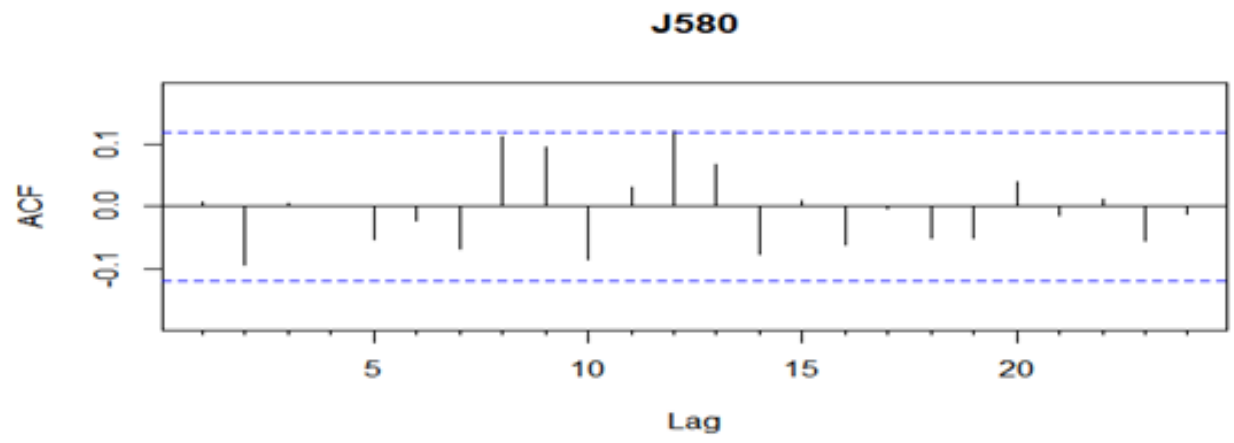

Figure 2. ACF diagram

The ACF in Figure 2 and the PACF in Figure 3 indicate that there are no significant auto correlations in the data.

The Box-Ljung test for auto-correlation of the monthly SA Financial Index (J580) return series was performed, giving a Chi-squared value $=0.015867, \mathrm{df}=1$ and a $\mathrm{p}$-value $=0.8998$.

The test revealed no presence of significant auto-correlation in the return distribution since the p-values are greater than 0.05 were obtained, indicating weak evidence against the null hypothesis, so we fail to reject the null hypothesis of no autocorrelation. This means that the return distribution is independently distributed.

\subsection{Analysing Losses and Gains}

The data set is now analysed after separating it into losses and gains. 


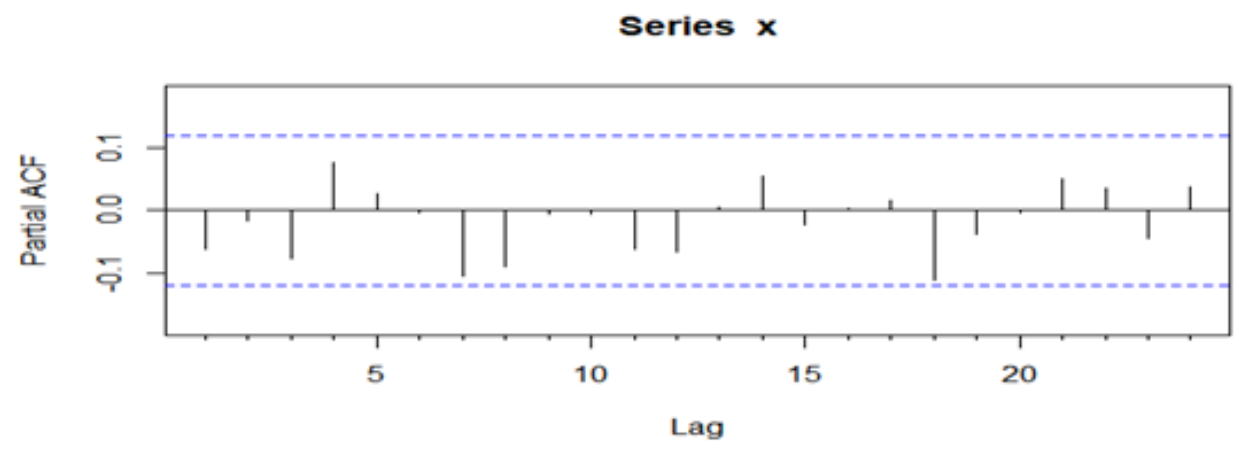

Figure 3. PACF diagram

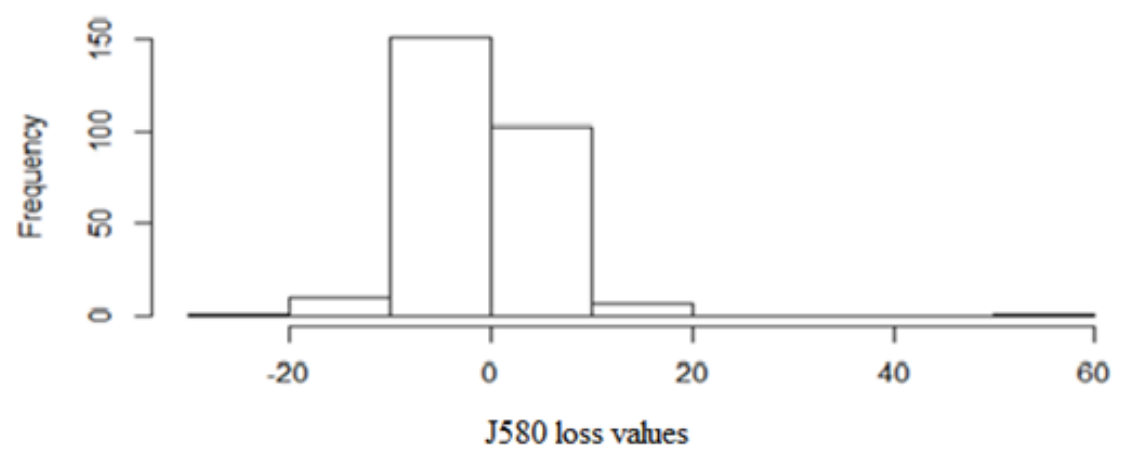

Figure 4. Histogram of the Loss values $L_{t}=-r_{t}$

The histogram in Figure 4 is on the return series $L_{t}$ and shows a heavy tail on the right tail of the histogram (right tail of the loss distribution/function, $L_{t}$ ). The Fretchet class (fat-tailed with $\xi>0$ ) distribution will most likely fit this tail. 


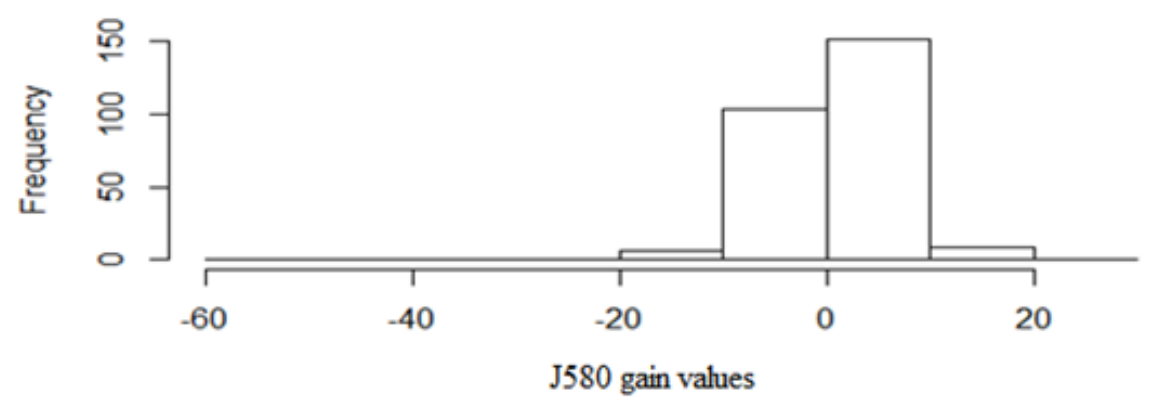

Figure 5. Histogram of the Gain values $r_{t}$

The histogram in Figure 5 is on the return series $r_{t}$ and shows a short tail on the right tail of the histogram (right tail of the return series distribution, $r_{t}$ ). The negative Weibull class (short-tailed with $\xi<0$ ) distribution will most likely fit this tail.

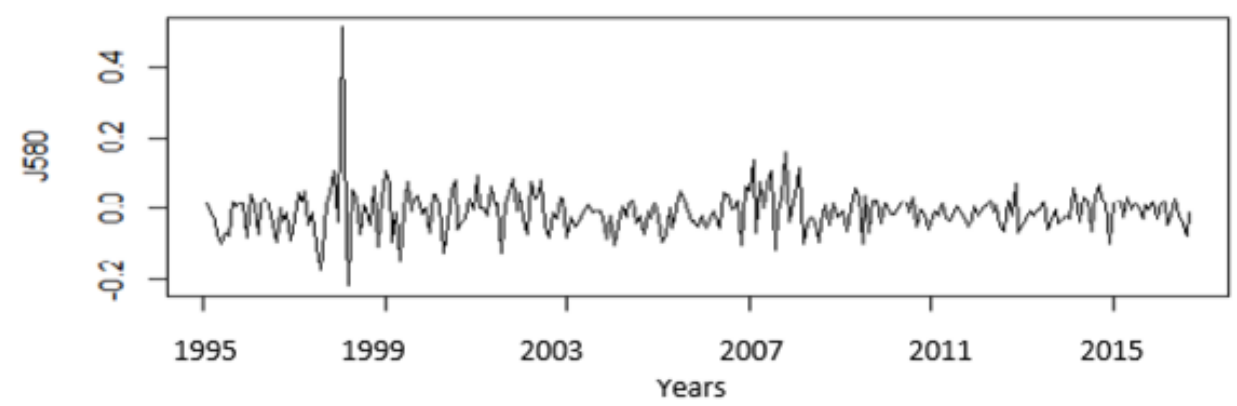

Figure 6. Time series plot of the $L_{t}=-r_{t}$, negative monthly returns of South African Financial Index (J580) from1995 to 2018, highlighting analysis in maximum losses.

The loss in 1997/1998 shows as a peak and will be a maximum point when using a loss function together with other losses in years such as 2007/2008.

The highest or extreme gains seem to occur soon after a stock market crash e.g in 1997-1998, 2007-2008 and 2015-2016. The $L_{t}$ monthly returns highlights analysis of losses. The $r_{t}$ monthly returns series highlights the analysis in mainly gains. Figure 6 and Figure 7 are mirror images of the same data set. However, one graph highlights losses and the other highlights gains as maxima. For both the losses and gains, the return series appear stable and that there is no presence of a unit root in the monthly data as confirmed by the Augmented Dickey-Fuller Test. It was concluded that data is stationary for both losses (when the series is inverted, i.e. forming the mirror image) and gains. The maximum loss and gain values are $0.5112(51.12 \%)$ and $0.2165(21.65 \%)$ respectively (see also Table 1).

In order to confirm the presence of the different stylised facts in the log return series, the descriptive statistics of the data set are below. 


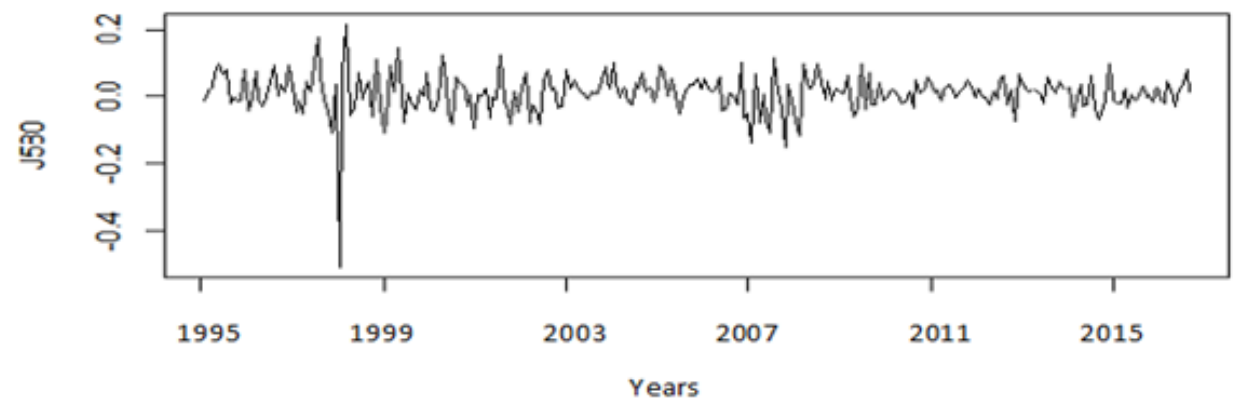

Figure 7. Time series plot of the $r_{t}$ monthly returns of South African Financial Index (J580) from 1995 to 2018 , highlighting analysis in maximum gains.

Table 1. Descriptive Statistics of the South African Financial Index (J580) returns for negative returns and positive returns.

\begin{tabular}{|c|c|c|}
\hline Period & Minima( Right tail of loss function, $L_{t}$ ) & Maxima( Right tail of $r_{t}$ ) \\
\hline Number of Observations & 91 & 91 \\
\hline Minimum & -0.067709 & -0.028955 \\
\hline Maximum & 0.511949 & 0.216516 \\
\hline Mean & 0.032407 & 0.051278 \\
\hline Kurtosis & 29.665063 & 1.494774 \\
\hline Skewness & 4.426538 & 0.845550 \\
\hline Standard Deviation & 0.065231 & 0.042924 \\
\hline
\end{tabular}

In Table 1, some characteristics of a financial time series for the minima returns such as positive skewness, and a large positive kurtosis are present in this data set. This allows us to infer that the return series is fat-tailed although kurtosis for the positive returns is less than 3 which violates the stylised financial fact that kurtosis should be greater than 3 . The maxima returns also exhibit positive kurtosis and positive skewness, which allows us to infer that the series is fat-tailed. The maximum loss found in the right tail of the loss function is $0.5112(51.12 \%)$ and the maximum gain in the left tail of the loss function is $0.2165(21.65 \%)$. The tails would be described in the opposite way if the return series $r_{t}$ is used instead of the loss function $L_{t}$.

\subsection{Fitting the Generalised Extreme Value Distribution using the block maxima method.}

The monthly return sample can give quarterly non-overlapping blocks, for the successive years: 1995 to 2018 . The minima/maxima returns extracted from the blocks constituted the data points, useful in fitting the GEVD. The estimated parameters help to calculate tail-related risk measures.

Figure 8 and Figure 9 shows the quarterly block minima and maxima data points (91 data points each for minima and maxima) from the South African Financial Index (J580). 


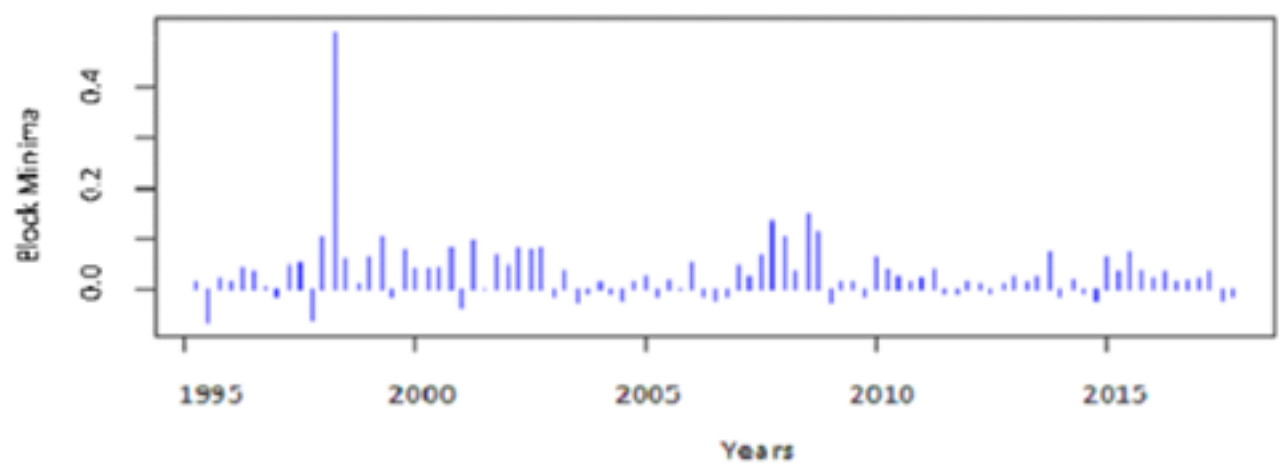

Figure 8. The quarterly block minima levels for South African Financial Index (J580) returns extracted from the right tail of the loss function $L_{t}$. (91 points only)

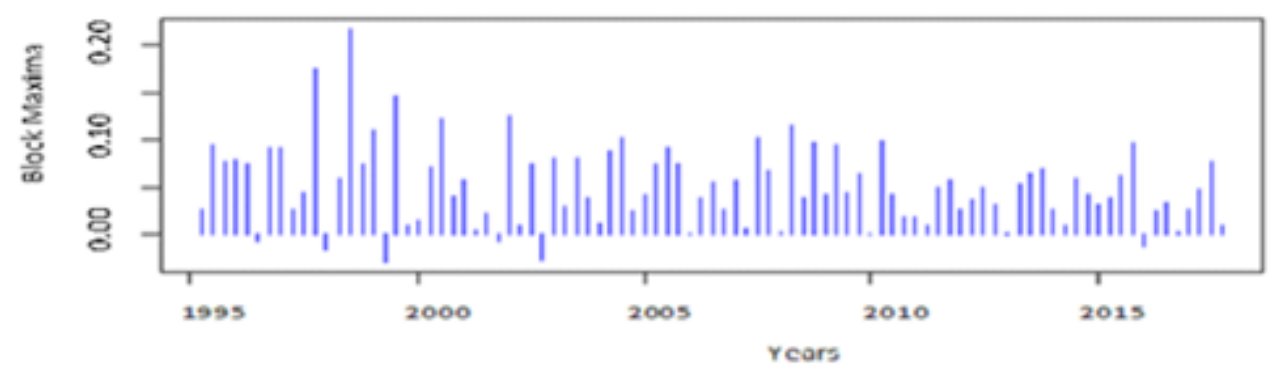

Figure 9. The quarterly block maxima levels for South African Financial Index (J580) extracted from the left tail of the loss function $L_{t}$. (91 points only)
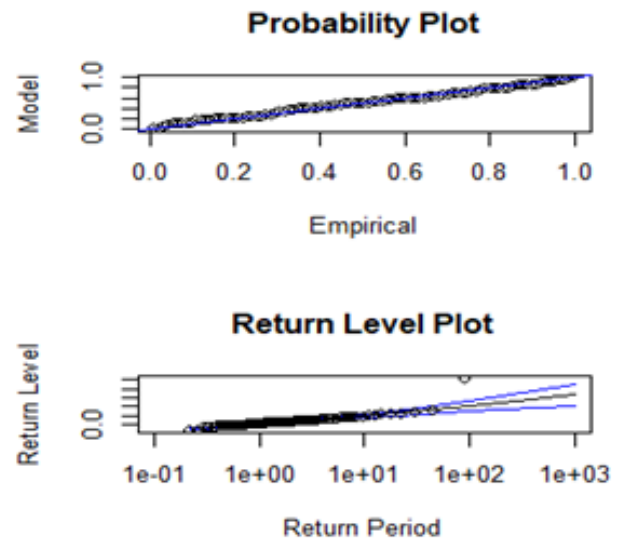
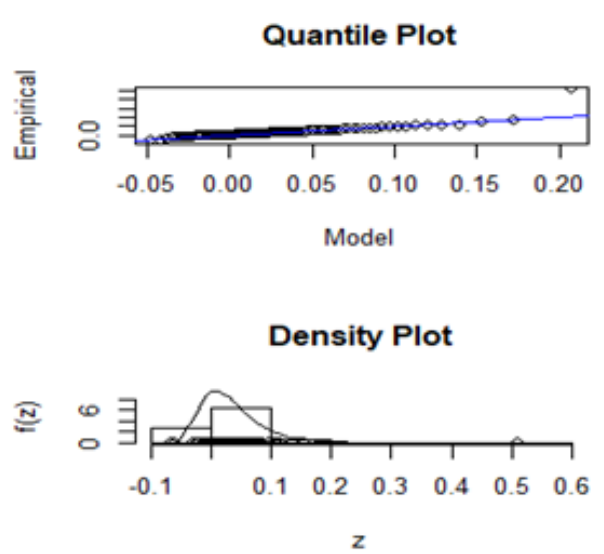

Figure 10. Probability, quantile, return level and density plots for the quarterly block minima of the return series (analysing the lower tail of $r_{t}$ or the right tail of $L_{t}$, losses).

The diagnostic plots assess the GEVD models for the quarterly minima and maxima. Figure 10, provides the diagnostic plots of the minima (losses) of the quarterly blocks to test for goodness of fit to the GEVD model. Data 

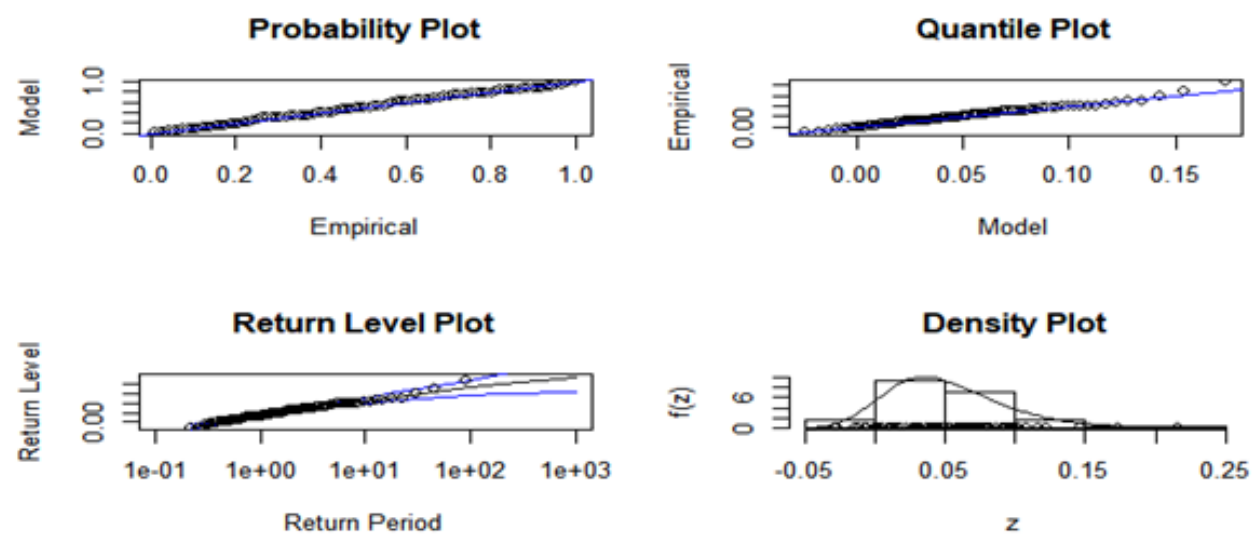

Figure 11. Probability, quantile, return level and density plots for the quarterly block maxima of the return series (analysing the upper tail of $r_{t}$ or the left tail of $L_{t}$, gains).

points in the QQ plot do not depart significantly from the straight line, the density plots confirms the conclusion obtained from the probability plots that the GEVD model provides a good model fit for the data at the tails of the distribution. The assumptions for fitting the GEVD to the data appear reasonable for the model. The diagnostic plots are in favour of the fitted model and the GEVD model is a good fit for the tails of the South African Financial Index (J580). The diagnostic plots for the maxima returns in Figure 11 are also in favour of the fitted model for the left tail of $L_{t}$ (gains) or the upper tail of $r_{t}$.

\subsection{Estimation of parameters and return levels (maximum potential loss) and potential gain}

Table 2 below shows the parameter estimates. The model parameters are useful in forecasting the return levels and their confidence intervals. The quarterly block minima/maxima index returns are useful to study the left and the right tails of the underlying distribution respectively.

The extreme value index parameter is not significant at very high confidence levels. The next table gives the parameter estimates at lower confidence levels. 
Table 2. Parameters and the return levels (point and interval estimates at $99 \%, 95 \%$ and $90 \%$ level of confidence) for 8 and 40 quarterly return periods for the right tail and left tail of the South African Financial Index (J580).

\begin{tabular}{|c|c|c|c|c|c|c|}
\hline & \multicolumn{3}{|c|}{ Right Tail of $L_{t}$ (Minima) } & \multicolumn{3}{|c|}{ Left Tail of $L_{t}$ (Maxima) } \\
\hline \multicolumn{7}{|c|}{$99 \%$ level of confidence } \\
\hline & Lower Bound & Point Estimate & Upper Bound & Lower Bound & Point Estimate & Upper Bound \\
\hline Shape Parameter, $\xi$ & $-0,0578$ & 0.0583 & 0.1784 & -0.2344 & -0.0674 & 0.0996 \\
\hline Scale Parameter, $\sigma$ & 0.0306 & 0.0385 & 0.0463 & 0.0286 & 0.0362 & 0.4381 \\
\hline Location Parameter, $\mu$ & -0.0030 & 0.0082 & 0.0194 & 0.0220 & 0.3278 & 0.0436 \\
\hline 2 years ( 8 quarters) & 0.0654 & 0.0904 & 0.1154 & 0.0822 & 0.1009 & 0.1197 \\
\hline 10 years (40 quarters) & 0.1146 & 0.1698 & 0.2173 & 0.1149 & 0.1506 & 0.1863 \\
\hline \multicolumn{7}{|c|}{$95 \%$ level of confidence } \\
\hline & Lower Bound & Point Estimate & Upper Bound & Lower Bound & Point Estimate & Upper Bound \\
\hline Shape Parameter, $\xi$ & -0.0300 & 0.0583 & 0.1466 & -0.1944 & -0.0674 & 0.0517 \\
\hline Scale Parameter, $\sigma$ & 0.0325 & 0.0385 & 0.0445 & 0.0304 & 0.0362 & 0.0420 \\
\hline Location Parameter, $\mu$ & -0.0003 & 0,0082 & 0.0167 & 0.0245 & 0.0328 & 0.0410 \\
\hline 2 years ( 8 quarters) & 0.0714 & 0.0904 & 0.1094 & 0.0887 & 0.1001 & 0.1152 \\
\hline 10 years (40 quarters) & 0.1269 & 0.1656 & 0.2051 & 0.1234 & 0.1506 & 0.1778 \\
\hline \multicolumn{7}{|c|}{$90 \%$ level of confidence } \\
\hline & Lower Bound & Point Estimate & Upper Bound & Lower Bound & Point Estimate & Upper Bound \\
\hline Shape Parameter, $\xi$ & -0.0158 & 0.0583 & 0.1324 & -0.1740 & -0.0674 & 0.0393 \\
\hline Scale Parameter, $\sigma$ & 0.0335 & 0.0385 & 0.0435 & 0.0313 & 0.0362 & 0.0411 \\
\hline Location Parameter, $\mu$ & 0.0010 & 0.0082 & 0.0154 & 0.0259 & 0.0328 & 0.0397 \\
\hline 2 years (8 quarters) & 0.0745 & 0.0904 & 0.1064 & 0.0889 & 0.1009 & 0.1129 \\
\hline 10 years (40 quarters) & 0.1332 & 0.1660 & 0.1988 & 0.1278 & 0.1506 & 0.1734 \\
\hline
\end{tabular}

Table 3. Parameters and the return levels (point and interval estimates at $85 \%, 80 \%, 75 \%$ and $70 \%$ level of confidence for 8 and 40 quarterly return periods for the right tail and left tail of the South African Financial Index (J580).

\begin{tabular}{|c|c|c|c|c|c|c|}
\hline & \multicolumn{3}{|c|}{ Right Tail of $L_{t}$ (Minima) } & \multicolumn{3}{|c|}{ Left Tail of $L_{t}$ (Maxima) } \\
\hline \multicolumn{7}{|c|}{$85 \%$ level of confidence } \\
\hline & Lower Bound & Point Estimate & Upper Bound & Lower Bound & Point Estimate & Upper Bound \\
\hline Shape Parameter, $\xi$ & -0.0066 & 0.0583 & 0.1232 & -0.1607 & -0.0674 & 0.0250 \\
\hline Scale Parameter, $\sigma$ & 0.0341 & 0.03848 & 0.0429 & 0.03192 & 0.0362 & 0.0404 \\
\hline Location Parameter, $\mu$ & 0.0026 & 0.0082 & 0.01378 & 0.0267 & 0.0328 & 0.0388 \\
\hline 2 years (8 quarters) & 0.0764 & 0.0904 & 0.1044 & 0.0904 & 0.1009 & 0.1114 \\
\hline 10 years (40 quarters) & 0.1373 & 0.1660 & 0.1947 & 0.1306 & 0.1506 & 0.1706 \\
\hline \multicolumn{7}{|c|}{$80 \%$ level of confidence } \\
\hline & Lower Bound & Point Estimate & Upper Bound & Lower Bound & Point Estimate & Upper Bound \\
\hline Shape Parameter, $\xi$ & 0.0005 & 0.0583 & 0.1161 & -0.1505 & -0.0674 & 0.0157 \\
\hline Scale Parameter, $\sigma$ & 0.0346 & 0.03848 & 0.0424 & 0.0324 & 0.0362 & 0.0400 \\
\hline Location Parameter, $\mu$ & 0.0026 & 0.0082 & 0.0138 & 0.0274 & 0.0328 & 0.0382 \\
\hline 2 years (8 quarters) & 0.0780 & 0.0904 & 0.0904 & 0.0916 & 0.1009 & 0.1102 \\
\hline 10 years (40 quarters) & 0.1404 & 0.1660 & 0.1915 & 0.1328 & 0.1506 & 0.1684 \\
\hline \multicolumn{7}{|c|}{$75 \%$ level of confidence } \\
\hline & Lower Bound & Point Estimate & Upper Bound & Lower Bound & Point Estimate & Upper Bound \\
\hline Shape Parameter, $\xi$ & 0.0065 & 0.0583 & 0.1101 & -0.1494 & -0.0674 & 0.0072 \\
\hline Scale Parameter, $\sigma$ & 0.0350 & 0.03848 & 0.0420 & 0.0328 & 0.0362 & 0.0396 \\
\hline Location Parameter, $\mu$ & 0.0032 & 0.0082 & 0.0132 & 0.0279 & 0.0328 & 0.0376 \\
\hline 2 years (8 quarters) & 0.0793 & 0.0904 & 0.1016 & 0.0925 & 0.1009 & 0.1093 \\
\hline 10 years (40 quarters) & 0.1430 & 0.1660 & 0.1890 & 0.1347 & 0.1506 & 0.1665 \\
\hline \multicolumn{7}{|c|}{$70 \%$ level of confidence } \\
\hline & Lower Bound & Point Estimate & Upper Bound & Lower Bound & Point Estimate & Upper Bound \\
\hline Shape Parameter, $\xi$ & 0.016 & 0.0583 & 0.1050 & -0.1350 & -0.0674 & -0.0001 \\
\hline Scale Parameter, $\sigma$ & 0.0353 & 0.0385 & 0.0416 & 0.0331 & 0.0362 & 0.0393 \\
\hline Location Parameter, $\mu$ & 0.0037 & 0.0082 & 0.0127 & 0.0284 & 0.0328 & 0.0371 \\
\hline 2 years (8 quarters) & 0.0804 & 0.0904 & 0.1005 & 0.0934 & 0.1010 & 0.1084 \\
\hline 10 years (40 quarters) & 0.1453 & 0.1660 & 0.1866 & 0.1362 & 0.1506 & 0.1650 \\
\hline
\end{tabular}

The parameters are significant at lower confidence levels when zero is not included in a confidence interval. For the right tail: at $80 \%$ level of confidence and lower, the shape, scale and location parameters are all significant. For 
the left tail: at $70 \%$ level of confidence and lower, the shape, scale and location parameters are all significant.

In Table 2 and Table 3, the parameter estimates and their corresponding confidence intervals are presented. The shape parameter for the right tail of $L_{t}$ is positive and insignificant at $99 \%, 95 \%, 90 \%$ and $85 \%$ level of confidence. However, it is significant at the $80 \%$ level of confidence since the interval does not include zero. The shape $(\hat{\xi})$, scale $(\hat{\sigma})$ and location $(\hat{\mu})$ parameter estimates for the right tail values (minima) are $\hat{\xi}=0.0583, \hat{\sigma}=0.0385$ and $\hat{\mu}=0.0082$ respectively. The minima of the South African Financial Index (J580) log return series follow the fat-tailed Fretchet class distribution with $\hat{\xi}>0$.

This implies that the prospect of potential extreme losses in the South African Financial Index (J580) is significant at the $80 \%$ level of confidence. It is prudent not to ignore such information. The minima are unbounded and losses can be very big.

In Table 2 and Table 3 the return level estimates and their corresponding confidence intervals for the return periods of 8 and 40 quarters are presented. For the two year return period ( 8 quarters), in the right tail of the loss function, the return level is $9.04 \%$, meaning that the maximum potential loss observed is $9.04 \%$ in one quarter out of two years ( 8 quarters) on average. The interpretation is the same for 40 quarterly return periods. This is used to predict the potential loss (return level) for the return period of 8 quarters ( 2 years) and 40 quarters (10 years) in advance.

For the maxima (left tail of the loss function), the parameters and their corresponding confidence intervals are also in Tables 2 and 3. The shape $(\hat{\xi})$, scale $(\hat{\sigma})$ and location $(\hat{\mu})$ parameters estimates are $\hat{\xi}=-0.0674, \hat{\sigma}=0.0362$ and $\hat{\mu}=0.0328$ respectively at the $70 \%$ level of confidence. The parameters are significant at the $70 \%$ level of confidence. This implies that the gains follow the negative Weibull class distribution since $\xi<0$. The maxima are upper bounded, meaning that gains are somewhat limited in the South African Financial Index (J580) $\log$ return series. The shape parameter is negative at the $70 \%$ level of confidence and is significant because the interval does not include zero. At higher levels of confidence: $99 \%, 95 \%, 90 \%$ and $85 \%, 80 \%$ and $75 \%$ the shape parameter is not significant since their intervals contain zero. The prospect of potential extreme gains in the South African Financial Index (J580) log return series is significant but there is a limit. This occurs at a much lower confidence level of $70 \%$, a level not to be ignored completely.

The return level estimates and their corresponding confidence intervals for 8 and 40 quarterly return periods are presented in Table 2 and Table 3. For a two year ( 8 quarters) return period, in the left tail of the loss function, the return level is $10.09 \%$. The maximum potential gains observable is $10.09 \%$ in one quarter out of two years ( 8 quarters) on average. The interpretation is the same for 40 quarterly return periods. This is used to predict the potential gain (return level) for the return period of 8 quarters ( 2 years) and 40 quarters (10 years) in advance. According to [3], the return levels (maximum potential loss/gain) is a stress index, the stress index is always a concern to investors and risk analysts as part of their risk management process.

Clearly, the right tail of the loss function (minima) is heavier than the left one (maxima). This is evident from the estimated value of the shape parameter, which is positive and significant in in the right tail, but negative in the left tail case at the $70 \%$ significance level. As a comparative analysis of the tail-related risk measures between the right tails and the left tails, from 1995 to 2018, the maximum potential losses (downside risk) is lower than the maximum potential gains (upside risk) in the short term ( 8 quarters). This is because estimated right tail (of $L_{t}$ ) return level of $9.04 \%$ is less than the left tail (of $L_{t}$ ) return level of $10.09 \%$. The maximum potential gain is lower than the maximum potential loss in the longer term (40 quarters) since the estimated left tail ( of $L_{t}$ ) return level of $15.06 \%$ is less than right tail (of $L_{t}$ ) return level of $16.60 \%$. This is not surprising since gains are limited but losses are not, for the given data set. The analysis is useful in helping investors prepare and protect equity portfolios from future extreme losses. 
We consider both the left and the right tail of the return distribution of the South African Financial Index (J580). The reason is that the left tail represents losses for an investor with a long position on the index, whereas the right tail represents losses for an investor being short on the index [22]. Estimation of future return levels for equity returns provides essential input to risk mitigating measures to reduce potential damage caused by foreign currency crises, credit defaults and stock market crashes.

\subsection{The Likelihood Ratio Test}

Although the Likelihood Ratio Test was carried out, the issue is, at what confidence level is the GEVD shape parameter significant.

The confidence intervals for the shape parameter of the right tail at 99\%, 95\%, 90\%, $85 \%$ and the left tail at $99 \%, 95 \%$ and $90 \%, 85 \%, 80 \%$ and $75 \%$ level of confidence contains a zero, which implies there is a possibility that the Gumbel class distribution may be a better fit, therefore a formal test based on the likelihood ratio test was carried out.

Null Hypothesis $H_{0}: \xi=0$

Alternate Hypothesis $H_{1}: \xi \neq 0$.

When the likelihood ratio test is less than the corresponding chi square critical value, we fail to reject null hypothesis which means that Gumbel family will be a good fit for our data. When the likelihood ratio test is greater than the corresponding chi square critical value, we reject null hypothesis which means that our data belong to either the Weibull class distribution or the Fretchet class according to the sign of the shape parameter. The model fits the Weibull class when shape parameter is negative and the Fretchet class when shape parameter is positive.

\section{The results obtained for the right tail at $95 \%$ level of confidence:}

The likelihood-ratio test for the significance on $\xi$ was performed.

The likelihood-ratio test statistic $=2.2657$, chi-square critical value $=3.8415, \alpha=0.0500$, Degrees of Freedom $=$ $1.0000, p$-value $=0.1323$. From the results, $\xi=0$, the Gumble class distribution is the appropriate distribution to fit.

\section{The results for obtained for the left tail at $95 \%$ level of confidence are indicated below:}

Likelihood-ratio $=0.91666$, chi-square critical value $=3.8415, \alpha=0.0500$, Degrees of Freedom $=1.0000, p$ value $=0.3384$

From the results, $\xi=0$, the Gumble class distribution is the appropriate distribution to fit.

The shape parameter for the right tail of $L_{t}$ is positive and observed to be insignificant at $99 \%, 95 \%, 90 \%$ and $85 \%$ level of confidence. The log return series follows the Gumble class distribution with $\hat{\xi}=0$ as revealed by the Likelihood Ratio Test at these levels of confidence interval which implies the parameter is insignificant. Similarly the shape parameter for the left tail is negative and insignificant at $99 \%, 95 \%, 90 \%, 85 \%, 80 \%$ and $75 \%$ level of significance. The log return series also follows the Gumble class distribution with $\hat{\xi}=0$ as revealed by the Likelihood Ratio Test at these levels of confidence intervals which also implies the parameter is insignificant.

So for the right tail, the parameter is significant at $80 \%$ level of significance and lower and for the left tail the parameter is significant at $70 \%$ level of confidence and lower.

When working with the GEVD it is not important to make this distinction and refit the Gumbel distribution since the GEVD combines the Fretchet, Weibull and Gumbel class distributions into one. 


\subsection{Return Level Estimation using the Generalised Pareto Distribution}

Parameters estimates for the GPD were arrived at using the maximum likelihood estimates. These parameters were used to estimate tail-related risk using return levels.

Table 4. Parameter estimation using MLE method for South African Financial Index (J580).

\begin{tabular}{|c|c|c|}
\hline & Right tail-downside risk (negative returns) & Left tail-upside risk (positive returns) \\
\hline & Threshold $\mu=0.04$ & Threshold $\mu=0.03$ \\
\hline Shape Parameter, $\xi$ & -0.06586863 & 0.28252147 \\
\hline Standard Error, $\epsilon$ & 0.110349819 & 0.153564442 \\
\hline Scale Parameter, $\sigma$ & 0.03609992 & 0.02688576 \\
\hline Standard Error, $\epsilon$ & 0.006108772 & 0.005400842 \\
\hline
\end{tabular}

The right tail follows the short-tailed negative Weibull family of distribution since $\xi<1$ and has a finite upper bound indicating an absolute maximum. The left tail has a positive shape parameter which is an indication that the left tail has fatter tails than the normal distribution, which can lead to the occurrence of extreme gains. The left tail follows the fat-tailed Fretchet class distribution. The South Africa's Johannesburg Stock Exchange All Share Index (ALSI) follows the short-tailed negative Weibull family distribution when focussing on extreme losses [9] which is consistent with the extreme losses (right tail) behaviour of the GPD in this study. [23] analysed FTSE/JSE Top 40 using the GPD. Their results revealed that the losses return distribution (right tail) follows the fat tailed Fretchet class distribution, which is inconsistent with the right tail in this study. These results are based on thresholds of $4 \%$ for the right tail and $3 \%$ for the left tail.

Table 5. GPD tail-related risk measures of return levels for South African Financial Index (J580).

\begin{tabular}{|c|c|c|c|}
\hline & Lower Bound & Point Estimate & Upper Bound \\
\hline \multicolumn{4}{|c|}{ Right Tail (Losses, Negative returns or Minima) } \\
\hline \multicolumn{4}{|c|}{ 95\% level of confidence } \\
\hline 2 years (8 quarters) & $14.99 \%$ & $16.01 \%$ & $21.28 \%$ \\
\hline 10 years (40 quarters) & $15.22 \%$ & $16.36 \%$ & $23.21 \%$ \\
\hline \multicolumn{3}{|c|}{ Left Tail (Gains, Positive Returns or Maxima) } \\
\hline \multicolumn{4}{|c|}{ 95\% level of confidence } \\
\hline 2 years (8 quarters) & $8.60 \%$ & $9.01 \%$ & $11.97 \%$ \\
\hline 10 years (40 quarters) & $8.71 \%$ & $9.15 \%$ & $13.04 \%$ \\
\hline
\end{tabular}

The estimated results of the tail-related risk measure using return levels for the GPD are shown in Table 5. The results indicate that for an investment on the South African Financial Index (J580), the possibility of losses (16.01\% in the short term and $16.36 \%$ in the long term) is greater than the possibility of gains $(9.01 \%$ in the short term and $9.15 \%$ in the long term) for a 2 year (short term) and 10 year (long term) periods respectively. The confidence intervals around these point estimates are also given in the table. [7], found that the exposure to extreme losses is higher than the possibility of extreme gains, which is consistent with results found in this study.

\subsection{Comparative analysis of the GEVD and the GPD return levels results}

In Table 6, the GEVD maximum potential losses (9.04\%) are lower than the maximum potential gains (10.10\%) in the short term (8 quarters). The maximum potential gains (15.06\%) are lower than the maximum potential losses $(16.56 \%)$ in the longer term (40 quarters). For the GPD, the maximum potential gains $(9.01 \%$ in the short term and $9.15 \%$ in the long term) are lower than the maximum potential losses $(16.01 \%$ in the short term and $16.36 \%$ in the long term).The GPD as a model however, has the problem that observations may not be independent. 
Table 6. Comparative analysis of the GEVD and the GPD return levels results

\begin{tabular}{|c|c|c|}
\hline Model & Generalised Extreme Value Distribution (GEVD) & Generalised Pareto Distribution (GPD) \\
\hline Return Period & \multicolumn{2}{|c|}{ Negative Returns/Right tail return level (losses) } \\
\hline & \multicolumn{2}{|c|}{ Maximum potential losses (\%) } \\
\hline 8 quarters & $9.04 \%$ & $16.01 \%$ \\
\hline 40 quarters & $16.56 \%$ & $16.36 \%$ \\
\hline Return Period & Positive Returns/Left Tail Return Level (gains) \\
\hline & \multicolumn{2}{|c|}{ Maximum potential gains (\%) } \\
\hline 8 quarters & $10.10 \%$ & $9.01 \%$ \\
\hline 40 quarters & $15.06 \%$ & $9.15 \%$ \\
\hline
\end{tabular}

\section{Conclusion and areas of further study}

\subsection{Conclusions}

The objective of this study was to model and extend the application of GEVD in quantifying tail-related risk measures of the South African Financial Index (J580) return series. Parameter estimates for the GEVD help to arrive at the maximum potential loss/gain of the South African Financial Index (J580) returns using return levels as a measure of tail-related risk. Logarithmic returns from the monthly South African Financial Index (J580) deviate from the normal distribution and are fat-tailed in nature. The EVT model is more appropriate to fit to the fat-tails of the distribution of monthly South African Financial Index (J580) return series. The monthly return series with quarterly block minima/maxima were fitted to the GEVD. The maximum likelihood method was used to estimate the parameters (shape, scale and location), and the return levels (maximum potential loss) for the right tail of the loss function and maximum potential gain, were calculated at selected return periods.

The diagnostic plots showed a good fit for the GEVD. The study reveals that losses follows the fat-tailed Fretchet class distribution and positive returns or gains follow the short-tailed negative Weibull class distribution. This implies loses can be very big but the gains are somewhat limited when investing in the South African Financial Index (J580). Conclusions are however made at lower confidence intervals.

The main findings of the study are:

i. for the GEVD the maximum potential losses are lower than the maximum potential gains in the short term and the maximum potential gains are lower than the maximum potential losses in the long term

ii. for the comparative GPD approach, the maximum potential losses are greater than the maximum potential gains both in the short and the long term.

Extending previous studies, the purpose of this study is to support investors with relevant information so that they can choose appropriate risk mitigating measures to reduce the potential damage arising from foreign exchange crises, large credit defaults and stock market crashes. The results suggest that EVT can be utilised effectively in estimating in advance, tail-related risk measures associated with return periods and return levels of the South African Financial Index (J580).

\subsection{Areas of further study}

Areas for further study would include the comparison of traditional methods for risk measures with the GEVD and GPD models. This is because traditional models don't take into account the instability of financial markets that cause extreme values. 
1. T. Rahman, Z. Hossain, and M. Habibullah, Stock Market Crash in Bangladesh: The Moneymaking Psychology of Domestic Investors, American Journal of Theoretical and Applied Business, vol. 3, no. 3, pp. 43, 2017, doi: 10.11648/j.ajtab.20170303.12.

2. S. Aboura, When the U.S. Stock Market Becomes Extreme?, Risks, vol. 2, no. 2, pp. 211225, 2014, doi: 10.3390/risks2020211.

3. G. Magnou, An application of extreme value theory for measuring financial risk in the Uruguayan Pension Fund COMPENDIUM, vol. 4, no. 7, pp. 119, 2017.

4. P. Yiou, et al. Nonlinear Processes in Geophysics Weather regime dependence of extreme value statistics for summer temperature and precipitation, Nonlinear Processes in Geophysics, vol. 15, pp. 365378, 2008.

5. C. Sigauke, M.R. Makhwiting, and M. Lesaoana, Modelling conditional heteroskedasticity in JSE stock returns using the Generalised Pareto Distribution, African Review of Economics and Finance, vol. 6, no. 1, pp. 4155, 2014.

6. H.M. Markowitz, Portfolio Selection, The Journal of Finance, vol. 7, no. 1, pp. 7791, 1952, doi:10.2307/2975974. JSTOR 2975974.

7. M. Gilli, and E. Kllezi, E. An application of extreme value theory for measuring financial risk, Computational Economics, vol. 27 , no. 23, pp. 207228, 2006, doi: 10.1007/s10614-006-9025-7.

8. D.E. Allen, A.K. Singh, and R.J. Powell, Extreme Market Risk - An Extreme Value Theory Approach, Edith Cowan University Publication, 2011.

9. M.R. Makhwiting, C. Sigauke, and M. Lesaoana, Modelling tail behaviour of returns using the generalised extreme value distribution, Economics, Management, and Financial Markets, vol. 9, no. 1, 2014.

10. A. Heymans, and L. Santana, How efficient is the Johannesburg Stock Exchange really?, South African Journal of Economic and Management Sciences, vol. 21, no. 1, 2018, a1968. https://doi. org/10.4102/sajems. v21i1.1968.

11. B.Y.A. Ferreira, and L. De Haan, On the block maxima method in extreme value theory: PWM estimators, Annals of Statistics, vol. 43, no. 1, pp. 276298, 2015, doi: 10.1214/14-AOS1280.

12. H. Hasan, N.F.A. Radi, and S. Kassim, Modeling the distribution of extreme share return in Malaysia using Generalized Extreme Value (GEV) distribution, AIP Conference Proceedings, 1450(May 2012), pp. 8289, 2012, doi: 10.1063/1.4724121.

13. N. Boudrissa, H. Cheraitia, and L. Halimi, Modelling maximum daily yearly rainfall in northern Algeria using generalized extreme value distributions from 1936 to 2009, Meteorological Applications, vol. 24, no. 1, pp. 114119, 2017, doi:10.1002/met.1610.

14. G. Lazoglou, and C. Anagnostopoulou, An Overview of Statistical Methods for Studying the Extreme Rainfalls in Mediterranean, Proceedings, Vol. 1, no. 5, pp. 681, 2017, doi: 10.3390/ecas2017-04132.

15. S. Coles, An Introduction to Statistical Modeling of Extreme Values. 3rd edn. Bristol: Springer, 2001.

16. A.F. Jenkinson, (1955) The Frequency Distribution of the Annual Maximum (or Minimum) of Meteorological Elements, Quarterly Journal of the Royal Meteorological Society, vol. 81, no. 348, pp. 158171, 1955.

17. B. Balkema, and L. deHaan, Residual lifetime at great age, Annals of Probability, Vol. 2, pp. 792-804, 1974.

18. J. Pickands, Statistical inference using extreme order statistics, Annals of Statistics, vol. 3, pp. 119-131, 1975.

19. R. Engle, Autoregressive Conditional Heteroscedasticity with Estimates of the Variance of United Kingdom Inflation, Econometrica, vol. 50, no. 4, pp. $9871007,1982$.

20. P. Hawkins, South Africas financial sector ten years on: performance since democracy Development Southern Africa, vol. 21 , no. 1, pp. 179204, 2004.

21. B. Butterworth, and S. Malherbe, For the Department of Trade and Industry, July 1999.

22. A. Mwamba, and T. Mhlanga, Extreme conditional value at risk: a coherent scenario for risk management, University of Johannesburg,2013.

23. D.C. Wentzel, and E. Mare, Extreme value theory An application to the South African equity market, Investment Analysts Journal, vol. 33, no. 66, pp. 73-77, 2007. 